\title{
Assessing left ventricular systolic function in shock: evaluation of echocardiographic parameters in intensive care
}

\author{
Lill Bergenzaun ${ }^{*}$, Petri Gudmundsson², Hans Öhlin ${ }^{3}$, Joachim Düring ${ }^{4}$, Anders Ersson ${ }^{4}$, Lilian Ihrman ${ }^{4}$,
} Ronnie Willenheimer ${ }^{5}$ and Michelle S Chew ${ }^{4}$

\begin{abstract}
Introduction: Assessing left ventricular (LV) systolic function in a rapid and reliable way can be challenging in the critically ill patient. The purpose of this study was to evaluate the feasibility and reliability of, as well as the association between, commonly used LV systolic parameters, by using serial transthoracic echocardiography (TTE).

Methods: Fifty patients with shock and mechanical ventilation were included. TTE examinations were performed daily for a total of 7 days. Methods used to assess LV systolic function were visually estimated, "eyeball" ejection fraction (EBEF), the Simpson single-plane method, mean atrioventricular plane displacement (AVPDm), septal tissue velocity imaging (TDIs), and velocity time integral in the left ventricular outflow tract (VTI).

Results: EBEF, AVPDm, TDIs, VTI, and the Simpson were obtained in 100\%, 100\%, 99\%, 95\% and 93\%, respectively, of all possible examinations. The correlations between the Simpson and EBEF showed $r$ values for all 7 days ranging from 0.79 to $0.95(P<0.01)$. the Simpson correlations with the other LV parameters showed substantial variation over time, with the poorest results seen for TDIs and AVPDm. The repeatability was best for VTI (interobserver coefficient of variation (CV) 4.8\%, and intraobserver CV, 3.1\%), and AVPDm (5.3\% and 4.4\%, respectively), and worst for the Simpson method (8.2\% and 10.6\%, respectively).

Conclusions: EBEF and AVPDm provided the best, and Simpson, the worst feasibility when assessing LV systolic function in a population of mechanically ventilated, hemodynamically unstable patients. Additionally, the Simpson showed the poorest repeatability. We suggest that EBEF can be used instead of single-plane Simpson when assessing LV ejection fraction in this category of patients. TDIs and AVPDm, as markers of longitudinal function of the LV, are not interchangeable with LV ejection fraction.
\end{abstract}

\section{Introduction}

Echocardiography is a useful tool for assessing cardiac function in hemodynamically unstable patients in the intensive care unit (ICU) [1]. For example, impairments of both systolic and diastolic left ventricular (LV) function have been observed in sepsis [2,3]. Only a few longitudinal transthoracic echocardiographic (TTE) [4-6] studies have been made of the LV systolic function in ICU patients with shock. Previous investigators have mainly concentrated on either acute changes $[3,7,8]$ or

\footnotetext{
* Correspondence: lill.bergenzaun@skane.se

'Department of Anaesthesiology, Institution of Clinical Sciences, Entrance 42, Skåne University Hospital, Lund University, Södra Förstadsgatan 101, S-20502 Malmö, Sweden

Full list of author information is available at the end of the article
}

used the transesophageal approach $[9,10]$. Serial evaluation of LV systolic function by echocardiography may provide additional insight into the relation between cardiac function and critical illness. A number of methods can be used to assess LV systolic function with echocardiography. These are well described in patients with cardiac disease and in healthy subjects, but few data exist from ICU patients. The different parameters do not necessarily assess the same features of LV systolic function. Their association to each other and how they change over time in the critically ill setting is unknown. LV ejection fraction (LVEF) can be assessed by the single- [11] or biplane [12] Simpson method, or more rapidly by visual estimation, "eyeball" ejection fraction

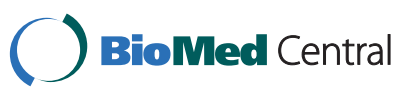


(EBEF) $[13,14]$. Although earlier studies have shown that satisfactory images can be obtained in only about $70 \%$ of patients with cardiac disease [15], or even less in ventilated patients [16], the development of second harmonic imaging [17] has improved endocardial border delineation, and the technique is today a standard feature in most modern ultrasound devices. Calculation of stroke volume (SV) in the LV outflow tract (LVOT) requires measurements of the velocity time integral (VTI) in LVOT and LVOT cross-sectional area. The latter can be difficult to obtain and is a known source of error in cardiac output (CO) measurement $[18,19]$. LVOT VTI, also called stroke distance, is feasible and reproducible in patients with cardiac disease [20] and is an accepted measure of LV function under changing hemodynamic conditions in experimental settings $[21,22]$. In patients with cardiac disease, left atrioventricular plane displacement (AVPD), also called mitral annulus movement, is a valuable tool for the assessment of longitudinal LV systolic function and is fairly easy to obtain [23,24]. The systolic pulsed tissue Doppler velocity of the LV septal wall (TDIs) is another new index of the longitudinal LV systolic function $[25,26]$. Both these parameters have been validated against LVEF measured by different methods in patients with cardiac disease $[27,28]$. In critically ill patients, tissue Doppler has predominantly been used in the assessment of LV diastolic function $[7,9]$ although recently also as a parameter for $\mathrm{LV}$ systolic function $[8,29]$. The aim of this study was to describe the feasibility, association between, and repeatability of, the different methods of evaluating LV systolic function in mechanically ventilated patients with shock, by using TTE.

\section{Materials and methods}

The study was approved by the Regional Ethics Review Board, Lund, Sweden (Dnr.187/2005). Informed consent was sought from the patient or, if not possible, from the next of kin. The study design was a single-center prospective observational cohort study of critically ill patients. Patients admitted to the mixed-bed ICU of Malmö University Hospital, Sweden, were screened for eligibility, and we included 55 consecutive patients with shock, defined as failure to maintain mean arterial pressure $\geq 70 \mathrm{~mm} \mathrm{Hg}$ despite adequate fluid resuscitation according to the surviving sepsis campaign algorithm [30]. All patients were older than 18 years, and all patients were mechanically ventilated. Exclusion criteria were pregnancy, known abnormalities of coagulation, fibrinolytic therapy, compromised immunity, or a "Do Not Attempt Resuscitation" order. Patients could be included only once. The study period was 7 days but was shorter in case of discharge from the ICU or death. Acute Physiology and Chronic Health Evaluation
(APACHE) II scores [31] were calculated at admission, and Sequential Organ Failure Assessment (SOFA) scores [32] were calculated for each day of the study period. After the initial resuscitation period, fluids were given at the treating clinician's discretion.

\section{Transthoracic echocardiography}

TTE examinations were performed within 12 hours of inclusion into the study. Subsequent studies were conducted daily for 7 days or until death or discharge from the ICU. The examinations were performed by either of four experienced echocardiographers (LB, MC, PG, $\mathrm{MD})$. Images were acquired by using a Hewlett-Packard Sonos 5500 (Andover, MA, U.S.A.) scanner and a 3$\mathrm{MHz}$ transthoracic transducer. Two-dimensional (2D) imaging examinations were performed in the standard apical four- and two-chamber views. Tissue harmonic imaging was used to enhance 2D image quality. These images were used to estimate LVEF by the EBEF and modified single-plane Simpson method. M-mode images were obtained at the LV septal, lateral, anterior, and posterior borders of the mitral ring [33] in the apical four- and two-chamber views, and an average AVPD value was calculated from these four locations (AVPDm). Pulsed-wave tissue Doppler recorded the peak systolic velocity (TDIs) of the LV septal wall at the level of the mitral annulus in the apical four-chamber view. The VTI of the LVOT was measured with pulsed wave Doppler in the apical five-chamber view. All TTE studies were recorded and measurements were taken in triplicate and averaged. Analysis of the measurements was made in Phillip's digital storing program Xcelera (Best, the Netherlands) offline. All analyses were made by one observer (LB). For repeatability studies, 15 randomly selected patients were re-analyzed after a twoyear interval to determine intraobserver repeatability (LB). In addition, another highly experienced investigator (PG) independently analyzed the images to determine interobserver repeatability.

\section{Statistical analysis}

Data are presented as median (lower quartile: upper quartile). Normality was tested for using the Kolmogorov-Smirnov test. For temporal changes, a repeatedmeasure ANOVA (Kruskall-Wallis test for nonparametric data) was applied. For correlation between two variables, the Spearman rank correlation was used, and for differences between two groups, the Mann-Whitney $U$ test was used. For comparison of paired values, a Wilcoxon signed-rank test was used, with a Bonferroni correction applied for multiple analyses. The intra- and interobserver variability was measured by the coefficient of variation $(\mathrm{CV})$. CV was defined as the ratio of the standard variation to the mean multiplied by 100 . 
Significance was set at $P<0.05$. The analyses were performed by using SPSS 17.0 for Windows.

\section{Results}

The original study included 55 consecutive patients. Two patients were excluded because of lack of written consent. One patient died 4 hours after study inclusion and before echocardiographic examination; one patient was morbidly obese and TTE was not possible; and one patient was incorrectly registered in the echocardiography database. These five patients were excluded from statistical analysis. Of 350 expected echocardiographic examinations, 91 were missing because of death or discharge from the ICU before Day 7 (25 of 50 patients). Another 28 examinations were lost during the installation of a new offline storage and analysis system. Thus, in total, 231 examinations were available for analysis. Patient characteristics are shown in Table 1. Two thirds of the population had septic shock. The remaining patients had shock due to other causes (pancreatitis, post-major noncardiac surgery, intoxication and multiorgan failure, gastrointestinal bleeding and portal hypertension, or unknown cause). Mechanical ventilations occurred in 45 (90\%) patients at inclusion and in all patients (100\%) during the first 2 days of the study period. Of 231 echocardiographic examinations, 227 (98\%) were performed with the patient mechanically ventilated. Pre-existing cardiac disease was present in $24 \%$ of patients, defined as severe arrhythmia, heart failure, or ischemic heart disease. In all, $48 \%$ had pre-existing treatment with $\beta$-blockers, ACE-inhibitors, Cachannel blockers, and/or nitrates. All patients were receiving vasopressors at inclusion.

\section{Assessment of feasibility}

Both EBEF and AVPDm could be assessed in 100\% of the investigations. TDIs and LVOT VTI could be

\section{Table 1 Patient characteristics}

\begin{tabular}{ll}
\hline Characteristic & Values \\
\hline Number of patients & 50 \\
Gender (M/F) & $36 / 14$ \\
Age (years) & $65(54: 74)$ \\
SOFA day 1 & $12(9: 14)$ \\
APACHE II & $24(19: 29)$ \\
ICU LOS & $8(4: 13)$ \\
Days on mechanical ventilation & $6.5(3: 13)$ \\
Vasopressor $\mu \mathrm{g} / \mathrm{kg} /$ min & $0.09(0.05: 0.14)$ \\
Cardiac disease (\%) & 24 \\
Preexisting therapy (\%) & 48 \\
ICU mortality (\%) & 24 \\
\hline
\end{tabular}

Data are presented as median (lower quartile:upper quartile). APACHE, Acute Physiology and Chronic Health Evaluation; ICU, intensive care unit; LOS, length of stay; SOFA, Sequential Organ Failure Assessment. Norepinephrine was used as vasopressor. Twelve patients received dobutamine, and one, adrenaline at inclusion. Ten patients received levosimendan during the study period. assessed in $99 \%$ and $95 \%$, respectively. In $7 \%$ of the investigations, the Simpson single-plane method could not be used to evaluate LVEF, because of poor image quality. On average, LV systolic function improved significantly over the 7-day observation period, as did SOFA score $(P<0.05)$. All measured LV systolic function parameters were significantly improved on day 6 compared with day 1 (Table 2). LV systolic function measurements on day 1 did not differ significantly between those who survived the 7-day study period and those who did not. Highly significant correlations were seen between the Simpson method and EBEF for all days $(r=0.794$ to $0.949 ; P<0.01)$. AVPDm, TDIs, and LVOT VTI correlated with the Simpson method, although these correlations were weaker than those seen between Simpson and EBEF (Table 3). The Simpson method showed considerable variations in correlations with AVPDm, TDIs, and LVOT VTI over the 7-day period. There were similar results for AVPDm versus TDIs, in which the correlation was reasonable at day $1(r=$ 0.427; $P<0.01)$ and on average for all measurements $(r$ $=0.439 ; P<0.01$ ), but varied considerably between days (Table 3).

\section{Repeatability of measurements}

Intraobserver repeatability ranged from $3.1 \%$ to $10.6 \%$, being worst for the Simpson single-plane method (Table 4). Similar findings were seen for interobserver repeatability (Table 4).

\section{Discussion}

The main findings of this study area follow:

1. Daily measurements of LV systolic function with EBEF, Simpson, AVDPm, LVOT VTI, and TDIs were all feasible in a group of hemodynamically unstable patients.

2. Serial measurements revealed that all LV systolic parameters improved significantly over time.

3. Good correlations were observed between EBEF and the Simpson single-plane method throughout the entire observation period. In contrast, poorer correlations were seen for Simpson versus AVPDm and Simpson versus TDIs, which varied substantially during the observation period.

4. An acceptable repeatability was found, with best results seen for LVOT VTI and AVPDm.

\section{Feasibility}

Our study demonstrated a good feasibility for all methods for the estimation of LV systolic function. Images were obtainable in $93 \%$ to $100 \%$, with the EBEF and AVPDm methods providing best results $(100 \%$ of examinations). This is somewhat surprising, given a commonly accepted view that TTE imaging is difficult 
Table 2 Systolic left ventricular function from Day 1 to Day 7

\begin{tabular}{|c|c|c|c|c|c|c|c|c|}
\hline & $\begin{array}{c}\text { Day } 1 \\
n=47\end{array}$ & $\begin{array}{c}\text { Day } 2 \\
n=44\end{array}$ & $\begin{array}{c}\text { Day } 3 \\
n=34\end{array}$ & $\begin{array}{c}\text { Day } 4 \\
n=31\end{array}$ & $\begin{array}{l}\text { Day } 5 \\
n=28\end{array}$ & $\begin{array}{l}\text { Day } 6 \\
n=26\end{array}$ & $\begin{array}{c}\text { Day } 7 \\
n=21\end{array}$ & $p$ value (over time) \\
\hline Simpson (\%) & $50(41: 58)$ & $53(45: 62)$ & $53(45: 58)$ & $56(48: 64)$ & $59(50: 63)$ & $62(57: 71)^{a}$ & $62(54: 65)$ & $<0.05$ \\
\hline EBEF (\%) & $49(40: 55)$ & $50(40: 65)$ & $50(40: 59)$ & $52(45: 63)$ & $58(45: 61)^{a}$ & $65(55: 70)^{a}$ & $65(50: 70)^{a}$ & $<0.05$ \\
\hline AVPDm (mm) & $10.5(8.0: 12.6)$ & $11(9.1: 12.6)$ & $11(9.0: 13.0)$ & $12(9.1: 14.0)$ & $11(9.9: 14.0)$ & $12(10.0: 14.8)^{a}$ & $12.5(11.0: 14.0)^{\mathrm{a}}$ & $<0.05$ \\
\hline TDIs (cm/sec) & $8.9(7.1: 10.0)$ & $9.1(7.6: 10.4)$ & $8.3(7.1: 10.0)$ & $9(7.4: 10.0)$ & $9.3(8.5: 11.0)$ & $10(8.9: 12.3)^{a}$ & 11 (9.0:13.5) & $<0.05$ \\
\hline LVOT VTI (cm) & $18(15: 23)$ & $20(17: 26)^{a}$ & $21(18: 24)^{a}$ & $21(18: 24)^{a}$ & $22(18: 24)^{a}$ & $22(20: 24)^{a}$ & $23(20: 24)$ & $<0.05$ \\
\hline SOFA & $12(9: 14)$ & $10(8: 13)^{a}$ & $8(6: 12)^{a}$ & $8(6: 11)^{a}$ & $7(5: 10)^{a}$ & $8(5: 9)^{a}$ & $7(5: 9)^{a}$ & $<0.05$ \\
\hline
\end{tabular}

Simpson, the Simpson single-plane method of the four-chamber view; AVPDm, atrioventricular plane displacement, mean value of septal, lateral, anterior, inferior measurements; EBEF, eyeball ejection fraction; LVOT VTI, velocity time integral in the left ventricular outflow tract; SOFA, Sequential Organ Failure Assessment score; TDIs, pulsed tissue Doppler imaging, systolic velocity of the septal portion of the mitral annulus. Data are presented as median (lower quartile:upper

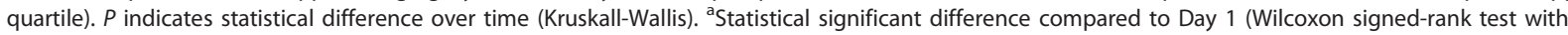
Bonferroni correction)

in mechanically ventilated patients. However, our results are in agreement with recent studies, in which the feasibility of acceptable TTE images in ICU patients was as high as $97 \%$ to $99 \%$ [34,35], with the apical view offering the best window [35]. From a clinical point of view, the excellent feasibility demonstrated in the present study supports the use of routine TTE, even in very ill, mechanically ventilated patients. This is a clear advantage, considering that TTE is considerably less invasive than transesophageal echocardiography.

\section{Systolic function over time}

Regardless of the method used, LV systolic function improved significantly over time, reflecting the clinical course of the patients. AVPDm and TDIs, as markers of the long-axis function of the LV, seemed to mirror clinical improvement, as did all other indices of LV systolic function. Post hoc analyses showed that all markers of LV systolic function were significantly improved on day 6 compared with day 1 , reflecting the clinical observation that most (44 of 50) patients were weaned of vasopressors and inotropes by day 6 .

\section{Correlations between the Simpson method and other methods}

The biplane Simpson method is a widely recommended method for the evaluation of LVEF [12]. Quantification of LVEF by the single-plane Simpson method correlates strongly with biplane Simpson in postinfarction patients and reliably detects small changes in LVEF [11]. EBEF can be performed more rapidly and has previously been shown to correlate well with more-formal quantitative assessments of LVEF, such as the Simpson single-plane and biplane EF [14], radionucleotide EF [13], and biplane contrast ventriculography [36] in patients with cardiac disease. EBEF is also independent of endocardial border tracing, which can be difficult in suboptimal images, an important limitation in ventilated patients. EBEF also integrates information about global and regional contractility, which may have advantages in a setting in which variations in regional contractility may reduce the accuracy of Simpson. Our study is in agreement with earlier studies showing good correlation between Simpson single-plane method and EBEF. We have further shown that this correlation is consistent throughout the entire observation period (Days 1 to 7). In contrast to EBEF, correlations between Simpson and

Table 3 Correlations $(r)$ of systolic parameters and level of significance $(P)$

\begin{tabular}{|c|c|c|c|c|c|c|c|c|c|c|c|c|c|c|c|c|}
\hline & \multicolumn{2}{|c|}{$\begin{array}{l}\text { Day } 1 \\
n=47\end{array}$} & \multicolumn{2}{|c|}{$\begin{array}{c}\text { Day } 2 \\
n=44\end{array}$} & \multicolumn{2}{|c|}{$\begin{array}{c}\text { Day } 3 \\
n=34\end{array}$} & \multicolumn{2}{|c|}{$\begin{array}{l}\text { Day } 4 \\
n=31\end{array}$} & \multicolumn{2}{|c|}{$\begin{array}{c}\text { Day } 5 \\
n=28\end{array}$} & \multicolumn{2}{|c|}{$\begin{array}{l}\text { Day } 6 \\
n=26\end{array}$} & \multicolumn{2}{|c|}{$\begin{array}{c}\text { Day } 7 \\
n=21\end{array}$} & \multicolumn{2}{|c|}{ All measurements $n=253$} \\
\hline & $r$ & $P$ & $r$ & $P$ & $r$ & $P$ & $r$ & $P$ & $r$ & $P$ & $r$ & $P$ & $r$ & $P$ & $r$ & $P$ \\
\hline Simpson vs. EBEF & 0.896 & a & 0.949 & a & 0.910 & a & 0.859 & a & 0.855 & a & 0.794 & a & 0.942 & a & 0.905 & a \\
\hline Simpson vs. AVPDm & 0.495 & a & 0.346 & b & 0.281 & & 0.392 & $b$ & 0.322 & & 0.101 & & 0.577 & a & 0.404 & a \\
\hline Simpson vs. TDls & 0.609 & a & 0.559 & a & 0.530 & a & 0.413 & $b$ & 0.152 & & 0.123 & & 0.310 & & 0.473 & a \\
\hline Simpson vs. LVOT VTI & 0.616 & a & 0.408 & a & 0.288 & & 0.499 & $b$ & 0.570 & a & 0.450 & $\mathrm{~b}$ & 0.742 & a & 0.513 & a \\
\hline AVPD vs. TDIs & 0.427 & a & 0.264 & & 0.456 & a & 0.356 & & 0.535 & a & 0.635 & a & 0.630 & a & 0.439 & a \\
\hline a & \multicolumn{16}{|c|}{ Corr sign at the 0.01 level. } \\
\hline b & \multicolumn{16}{|c|}{ Corr sign at the 0.05 level. } \\
\hline
\end{tabular}

Simpson single-plane method of the four-chamber view. AVPDm, atrioventricular plane displacement, mean value of septal, lateral, anterior, inferior measurements; EBEF, eyeball ejection fraction; LVOT VTI, velocity time integral in the left ventricular outflow tract; TDIs, pulsed tissue Doppler imaging, systolic velocity of the septal portion of the mitral annulus; Spearman rank correlation was used, and for differences between two groups, Mann-Whitney $U$ test was used. $P$ values have been corrected by the Bonferroni method. 
Table 4 Reproducibility of measurements

\begin{tabular}{cccccc}
\hline & EBEF & Simpson & TDIs & AVPDm & LVOT VTI \\
\hline Intraobserver & 6.8 & 10.6 & 8.2 & 4.4 & 3.1 \\
Interobserver & 9.9 & 8.2 & 7.2 & 5.3 & 4.8 \\
\hline
\end{tabular}

Intra- and interobserver coefficients of variation (\%) for EBEF; the Simpson single-plane method of the four-chamber view; AVPDm, atrioventricular plane displacement, mean value of septal, lateral, anterior, inferior measurements; $E B E F$, eyeball ejection fraction; LVOT VTI, velocity time integral in the left ventricular outflow tract; TDIs, pulsed tissue Doppler imaging, systolic velocity of the septal portion of the mitral annulus.

AVPDm, Simpson and TDIs, and even the correlations between AVPDm and TDIs varied substantially over the observation period. Previous studies in patients with cardiac disease have shown varying correlations between LVEF measured by radionucleotide or Simpson and AVPD or TDI, ranging from good [37,38] to poor $[39,40]$. In healthy subjects, the correlations were rather poor $[26,38,41,42]$.

AVPD and TDI may be superior in detecting subtle abnormalities of LV systolic function [43] and in patients with subclinical heart disease [44,45], as well as with increasing age [46]; longitudinal contractility is reduced whereas LVEF is preserved. A number of possible mechanisms exist by which subendocardial, longitudinal LV function may become impaired earlier than subepicardial, circumferential LV function [47]. Our observations and several previous findings emphasize that neither AVPD $[39,43,46]$ nor TDI [25] is interchangeable with LVEF, and by using LVEF solely when describing LV systolic function, the long-axis function of the heart is not necessarily considered. We found only moderate associations between AVPDm and TDIs, and they may also not be interchangeable, although TDI correlated well with AVPD in healthy individuals $[26,48]$. Several possible reasons may explain a lack of correlation between TDI and AVPD [23,49,50].

We showed that LVOT VTI was easily accessible and had a good intra- and interobserver repeatability. This is in line with previous studies in patients with aortic valve stenosis [51] and in other patients with cardiac disease [20]. Of note, animal studies have shown that LVOT VTI is an effective and noninvasive method when assessing LV systolic function under conditions of varying preload, heart rate, and inotropic state [21,22,52], an important asset in hemodynamically unstable patients. LVOT VTI is thought to be a better indicator of LV systolic function than is stroke volume, without the confounding factor of LVOT-area measurements [18,19,51]. This is especially true for ICU patients for whom usable images in the transthoracic parasternal view (used to measure LVOT diameter) can be obtained in about two thirds of patients [34].

\section{Repeatability}

The Simpson method showed the worst repeatability, which is comparable with the results of Lamia et al. in critically ill patients [53]. The repeatability using the EB technique was comparable. In contrast, we found good intra- and interobserver repeatabilities for LVOT VTI measurements. For the measurements of longitudinal LV systolic motion, we found clinically acceptable repeatability. We believe that these findings are clinically relevant, because the Simpson is the commonly recommended method for LV systolic function assessment, yet provided the poorest repeatability in this study.

\section{Limitations}

The influence of vasopressors, inotropes, and mechanical ventilation on echocardiographic measurements is uncertain. We have not excluded patients with previous known heart failure or atrial fibrillation, nor have we excluded patients with onset of atrial fibrillation during their critical illness, which might have influenced our results. Our intention was to investigate echocardiographic parameters that are well established in patients with cardiac disease, but less investigated in critically ill, mechanically ventilated patients with shock. We could have used contrast agents to facilitate the endocardial border detection, but this was not advisable at the time of data collection [54]. The strength of this study is the temporal approach and the simultaneous comparisons of five different, commonly used methods for the evaluation of LV systolic function. We found good feasibility and clinically acceptable reproducibility for our measurements, but all studies were conducted by experienced operators only. Echocardiography is user dependent, and results may be poorer in less-experienced hands.

\section{Conclusions}

Measurement of LV systolic function was feasible in hemodynamically unstable, mechanically ventilated patients. The different methods for the assessment of LV systolic function all reflected improvement in clinical state over time. However, the different methods did not all correlate well to one another, probably because they measure different components of LV systolic function and presumably do not change uniformly over time. EBEF and AVPDm provided the best, and Simpson, the worst feasibility. Additionally, Simpson showed the poorest repeatability. We suggest that EBEF can be used instead of single-plane Simpson when assessing LVEF and that combining different left ventricular indices, like EBEF and AVPD, when measuring LV systolic function, might be valuable in this category of patients. 


\section{Key messages}

- Assessment of left ventricular (LV) systolic function by transthoracic echocardiography (TTE) using five different echocardiographic parameters over a 7day period in ventilated patients with shock.

- All parameters were easy obtainable (93\% to 100\%) and had acceptable repeatability (coefficient of variation, $3.1 \%$ to $10.6 \%$ ); their correlation to one another showed substantial variation $(r=0.101$ to 0.949$)$ over time.

- Eyeball ejection fraction (EBEF) can be used instead of single-plane Simpson when assessing LV ejection fraction, but markers of the LV longitudinal function, such as tissue Doppler and atrioventricular plane displacement, are not interchangeable with LV ejection fraction.

\section{Abbreviations}

AVPDm: atrioventricular plane displacement mean; CO: cardiac output; CV: coefficient of variation; EBEF: eyeball ejection fraction; EF: ejection fraction; ICU: intensive care unit; LV: left ventricular; LVOT: left ventricular outflow tract; TDls: tissue Doppler imaging septal; TTE: transthoracic echocardiography; VTl: velocity time imaging.

\section{Acknowledgements}

The authors thank Magnus Dencker for critically reviewing the manuscript, and Nuray Güner, for statistical advice. The study was supported by grants from the Acta Foundation and the Region Skane County Council, Sweden. None of the funding agents was involved in study design, data collection, analysis and interpretation, or in writing and submitting the manuscript.

\section{Author details}

'Department of Anaesthesiology, Institution of Clinical Sciences, Entrance 42, Skåne University Hospital, Lund University, Södra Förstadsgatan 101, S-20502 Malmö, Sweden. ${ }^{2}$ Department of Biomedical Science, Malmö University, Södra Förstadsgatan 101, S- 20506 Malmö, Sweden. ${ }^{3}$ Department of Cardiology, Institution of Clinical Sciences, Skåne University Hospital, Lund University, Getingevägen 4, S- 22185 Lund, Sweden. ${ }^{4}$ Department of Intensive Care Medicine, Institution of Clinical Sciences, Entrance 42, Skåne University Hospital, Lund University, Södra Förstadsgatan 101, S-20502 Malmö, Sweden. ${ }^{5}$ Heart Health Group, Lund University, Geijersg. 4C, 21618 Limhamn, Sweden.

\section{Authors' contributions}

LB made substantial contributions to the conception and design of the study, participated in interpretation of data, made substantial contributions in acquisition and analysis of data, and helped to draft the manuscript. RW and $\mathrm{AE}$ made substantial contributions to conception and design of the study. MC made substantial contributions to conception and design of the study, participated in interpretation of data, and helped to draft the manuscript. PG made substantial contributions to conception and design of the study and made substantial contributions to acquisition and analysis of data. JD made substantial contributions to conception and design of the study and made contributions to the acquisition of data. $\mathrm{HO}$ participated in interpretation of data and helped to draft the manuscript. LI made contributions to the acquisition of data. All authors have made substantial intellectual contributions to the manuscript and have given final approval of the version to be published.

\section{Competing interests}

The authors declare that they have no competing interests.

Received: 14 April 2011 Revised: 13 June 2011

Accepted: 16 August 2011 Published: 16 August 2011

\section{References}

1. Cholley BP, Vieillard-Baron A, Mebazaa A: Echocardiography in the ICU: time for widespread use! Intensive Care Med 2006, 32:9-10.

2. Parker MM, Shelhamer JH, Bacharach SL, Green MV, Natanson C, Frederick TM, Damske BA, Parrillo JE: Profound but reversible myocardial depression in patients with septic shock. Ann Intern Med 1984, 100:483-490.

3. Poelaert J, Declerck C, Vogelaers D, Colardyn F, Visser CA: Left ventricular systolic and diastolic function in septic shock. Intensive Care Med 1997, 23:553-560

4. Jardin F, Brun-Ney D, Auvert B, Beauchet A, Bourdarias J: Sepsis-related cardiogenic shock. Crit Care Med 1990, 18:1055-1060.

5. Jardin F, Fourme T, Page B, Loubieres Y, Vieillard-Baron A, Beauchet A, Bourdarias JP: Persistent preload defect in severe sepsis despite fluid loading: a longitudinal echocardiographic study in patients with septic shock. Chest 1999, 116:1354-1359.

6. McLean AS, Huang SJ, Hyams S, Poh G, Nalos M, Pandit R, Balik M, Tang B, Seppelt I: Prognostic values of B-type natriuretic peptide in severe sepsis and septic shock. Crit Care Med 2007, 35:1019-1026.

7. Sturgess DJ, Marwick TH, Joyce CJ, Jones M, Venkatesh B: Tissue Doppler in critical illness: a retrospective cohort study. Crit Care 2007, 11:R97.

8. Sturgess DJ, Marwick TH, Joyce C, Jenkins C, Jones M, Masci P, Stewart D, Venkatesh B: Prediction of hospital outcome in septic shock: a prospective comparison of tissue Doppler and cardiac biomarkers. Crit Care 2010, 14:R44.

9. Bouhemad B, Nicolas-Robin A, Arbelot C, Arthaud M, Feger F, Rouby J-J: Isolated and reversible impairment of ventricular relaxation in patients with septic shock. Crit Care Med 2008, 36:766-774.

10. Vieillard-Baron A, Caille V, Charron C, Belliard G, Page B, Jardin F: Actual incidence of global left ventricular hypokinesia in adult septic shock. Crit Care Med 2008.

11. St John Sutton M, Otterstat JE, Plappert T, Parker A, Sekarski D, Keane MG, Poole-Wilson P, Lubsen K: Quantitation of left ventricular volumes and ejection fraction in post-infarction patients from biplane and single plane two-dimensional echocardiograms: a prospective longitudinal study of 371 patients. Eur Heart J 1998, 19:808-816.

12. Lang RM, Bierig M, Devereux RB, Flachskampf FA, Foster E, Pellikka PA, Picard MH, Roman MJ, Seward J, Shanewise JS, Solomon SD, Spencer KT, Sutton MS, Stewart WJ: Recommendations for chamber quantification: a report from the American Society of Echocardiography's Guidelines and Standards Committee and the Chamber Quantification Writing Group, developed in conjunction with the European Association of Echocardiography, a branch of the European Society of Cardiology. J Am Soc Echocardiogr 2005, 18:1440-1463.

13. Jensen-Urstad K, Bouvier F, Hojer J, Ruiz H, Hulting J, Samad B, Thorstrand C, Jensen-Urstad M: Comparison of different echocardiographic methods with radionuclide imaging for measuring left ventricular ejection fraction during acute myocardial infarction treated by thrombolytic therapy. Am J Cardiol 1998, 81:538-544.

14. Gudmundsson P, Rydberg E, Winter R, Willenheimer R: Visually estimated left ventricular ejection fraction by echocardiography is closely correlated with formal quantitative methods. Int I Cardiol 2005, 101:209-212.

15. Lang RM, Mor-Avi V, Zoghbi WA, Senior R, Klein AL, Pearlman AS: The role of contrast enhancement in echocardiographic assessment of left ventricular function. Am J Cardiol 2002, 90:28-34.

16. Vignon $P$, Mentec $H$, Terre $S$, Gastinne H, Gueret $P$, Lemaire F: Diagnostic accuracy and therapeutic impact of transthoracic and transesophageal echocardiography in mechanically ventilated patients in the ICU. Chest 1994, 106:1829-1834.

17. Nixdorff U, Matschke C, Winklmaier M, Flachskampf FA, Ludwig J, Pohle K, Land M, Gefeller O, Daniel WG: Native tissue second harmonic imaging improves endocardial and epicardial border definition in dobutamine stress echocardiography. Eur J Echocardiogr 2001, 2:52-61.

18. Minners J, Allgeier M, Gohlke-Baerwolf C, Kienzle RP, Neumann FJ, Jander N: Inconsistent grading of aortic valve stenosis by current guidelines: haemodynamic studies in patients with apparently normal left ventricular function. Heart 2010, 96:1463-1468.

19. Chew MS, Poelaert J: Accuracy and repeatability of pediatric cardiac output measurement using Doppler: 20-year review of the literature. Intensive Care Med 2003, 29:1889-1894. 
20. Thomas DE, Yousef ZR, Fraser AG: A critical comparison of echocardiographic measurements used for optimizing cardiac resynchronization therapy: stroke distance is best. Eur J Heart Fail 2009, 11:779-788.

21. Wallmeyer K, Wann LS, Sagar KB, Kalbfleisch J, Klopfenstein HS: The influence of preload and heart rate on Doppler echocardiographic indexes of left ventricular performance: comparison with invasive indexes in an experimental preparation. Circulation 1986, 74:181-186.

22. Sohn S, Kim HS, Han JJ: Doppler flow velocity measurement to assess changes in inotropy and afterload: a study in healthy dogs. Echocardiography 2002, 19:207-213.

23. Willenheimer R, Cline C, Erhardt L, Israelsson B: Left ventricular atrioventricular plane displacement: an echocardiographic technique for rapid assessment of prognosis in heart failure. Heart 1997, 78:230-236.

24. Alam M: The atrioventricular plane displacement as a means of evaluating left ventricular systolic function in acute myocardial infarction. Clin Cardiol 1991, 14:588-594.

25. Nikitin NP, Witte KKA: Application of tissue Doppler imaging in cardiology. Cardiology 2004, 101:170-184.

26. Alam M, Wardell J, Andersson E, Samad BA, Nordlander R: Characteristics of mitral and tricuspid annular velocities determined by pulsed wave Doppler tissue imaging in healthy subjects. J Am Soc Echocardiogr 1999, 12:618-628.

27. Pai RG, Bodenheimer MM, Pai SM, Koss JH, Adamick RD: Usefulness of systolic excursion of the mitral anulus as an index of left ventricular systolic function. Am J Cardiol 1991, 67:222-224.

28. Alam M, Hoglund C, Thorstrand C: Longitudinal systolic shortening of the left ventricle: an echocardiographic study in subjects with and without preserved global function. Clin Physiol 1992, 12:443-452.

29. Ikonomidis I, Nikolaou M, Dimopoulou I, Paraskevaidis I, Lekakis J, Mavrou I, Tzanela M, Kopterides P, Tsangaris I, Armaganidis A, Kremastinos DT: Association of left ventricular diastolic dysfunction with elevated NTpro-BNP in general intensive care unit patients with preserved ejection fraction: a complementary role of tissue Doppler imaging parameters and NT-pro-BNP levels for adverse outcome. Shock 2010, 33:141-148.

30. Dellinger RP, Carlet JM, Masur H, Gerlach H, Calandra T, Cohen J, GeaBanacloche J, Keh D, Marshall JC, Parker MM, Ramsay G, Zimmerman JL, Vincent $J$, Levy MM: Surviving Sepsis Campaign guidelines for management of severe sepsis and septic shock. Crit Care Med 2004, 32:858-873.

31. Knaus WA, Draper EA, Wagner DP, Zimmerman JE: Prognosis in acute organ-system failure. Ann Surg 1985, 202:685-693.

32. Vincent $\mathrm{J}$, de Mendonca A, Cantraine F, Moreno R, Takala J, Suter PM, Sprung CL, Colardyn F, Blecher S: Use of the SOFA score to assess the incidence of organ dysfunction/failure in intensive care units: results of a multicenter, prospective study: working group on "sepsis-related problems" of the European Society of Intensive Care Medicine. Crit Care Med 1998, 26:1793-1800.

33. Höglund C, Alam M, Thostrand C: Atrioventricular valve plane displacement in healthy persons. Acta Med Scand 1988, 224:557-562.

34. Jensen MB, Sloth E, Larsen KM, Schmidt MB: Transthoracic echocardiography for cardiopulmonary monitoring in intensive care. Eur J Anaesthesiol 2004, 21:700-707.

35. Jakobsen CJ, Torp P, Sloth E: Perioperative feasibility of imaging the heart and pleura in patients with aortic stenosis undergoing aortic valve replacement. Eur J Anaesthesiol 2007, 24:589-595.

36. Mueller X, Stauffer JC, Jaussi A, Goy JJ, Kappenberger L: Subjective visual echocardiographic estimate of left ventricular ejection fraction as an alternative to conventional echocardiographic methods: comparison with contrast angiography. Clin Cardiol 1991, 14:898-902.

37. Emilsson $\mathrm{K}$, Alam M, Wandt B: The relation between mitral annulus motion and ejection fraction: a nonlinear function. J Am Soc Echocardiogr 2000, 13:0896-0901.

38. Duzenli MA, Ozdemir K, Aygul N, Altunkeser BB, Zengin K, Sizer M: Relationship between systolic myocardial velocity obtained by tissue Doppler imaging and left ventricular ejection fraction: systolic myocardial velocity predicts the degree of left ventricular dysfunction in heart failure. Echocardiography 2008, 25:856-863.

39. Wandt B, Bojo L, Tolagen K, Wranne B: Echocardiographic assessment of ejection fraction in left ventricular hypertrophy. Heart 1999, 82:192-198.
40. Nikitin NP, Witte KK, Clark AL, Cleland JG: Color tissue Doppler-derived long-axis left ventricular function in heart failure with preserved global systolic function. Am J Cardio/ 2002, 90:1174-1177.

41. Carlhall CJ, Lindstrom L, Wranne B, Nylander E: Atrioventricular plane displacement correlates closely to circulatory dimensions but not to ejection fraction in normal young subjects. Clin Physiol 2001, 21:621-628.

42. Nikitin NP, Witte KK, Thackray SD, de Silva R, Clark AL, Cleland JG: Longitudinal ventricular function: normal values of atrioventricular annular and myocardial velocities measured with quantitative twodimensional color Doppler tissue imaging. J Am Soc Echocardiogr 2003, 16:906-921.

43. Yip G, Wang M, Zhang Y, Fung JW, Ho PY, Sanderson JE: Left ventricular long axis function in diastolic heart failure is reduced in both diastole and systole: time for a redefinition? Heart 2002, 87:121-125.

44. Fang $Z Y$, Leano R, Marwick TH: Relationship between longitudinal and radial contractility in subclinical diabetic heart disease. Clin Sci (Lond) 2004, 106:53-60.

45. Henein MY, Gibson DG: Long axis function in disease. Heart 1999, 81:229-231.

46. Emilsson $\mathrm{K}$, Wandt $\mathrm{B}$ : The relation between mitral annulus motion and ejection fraction changes with age and heart size. Clin Physiol 2000, 20:38-43.

47. Vinereanu D, Florescu N, Sculthorpe N, Tweddel AC, Stephens MR, Fraser AG: Differentiation between pathologic and physiologic left ventricular hypertrophy by tissue Doppler assessment of long-axis function in patients with hypertrophic cardiomyopathy or systemic hypertension and in athletes. Am J Cardiol 2001, 88:53-58.

48. Study Group of Echocardiography of the Italian Society of Cardiology, Mondillo S, Galderisi M, Ballo P, Marino PN: Left ventricular systolic longitudinal function: comparison among simple M-mode, pulsed, and M-mode color tissue doppler of mitral annulus in healthy individuals. $J$ Am Soc Echocardiogr 2006, 19:1085-1091.

49. Ballo P, Bocelli A, Motto A, Mondillo S: Concordance between M-mode, pulsed tissue Doppler, and colour tissue Doppler in the assessment of mitral annulus systolic excursion in normal subjects. Eur J Echocardiogr 2008, 9:748-753.

50. Chen QM, Li W, O'Sullivan C, Francis DP, Gibson D, Henein MY: Clinical in vivo calibration of pulse wave tissue Doppler velocities in the assessment of ventricular wall motion: a comparison study with $\mathrm{M}$ mode echocardiography. Int J Cardiol 2004, 97:289-295.

51. Geibel A, Gornandt L, Kasper W, Bubenheimer P: Reproducibility of Doppler echocardiographic quantification of aortic and mitral valve stenoses: comparison between two echocardiography centers. Am J Cardiol 1991, 67:1013-1021.

52. Saeian K, Wann LS, Sagar KB: Doppler echocardiographic evaluation of left ventricular function. Echocardiography 1990, 7:21-25.

53. Lamia B, Teboul J-L, Monnet X, Richard C, Chemla D: Relationship between the tricuspid annular plane systolic excursion and right and left ventricular function in critically ill patients. Intensive Care Med 2007, 33:2143-2149.

54. FDA: BMS' ultrasound drug Definity gets black box warning. FDAnews Drug Daily Bulletin 2007, 4:203.

doi:10.1186/cc10368

Cite this article as: Bergenzaun et al: Assessing left ventricular systolic function in shock: evaluation of echocardiographic parameters in intensive care. Critical Care 2011 15:R200. 